\title{
Case Report: Ectopic Odontoma of the Nasal Cavity
}

\author{
Brandon Musgrave, Robert Stapp, Frank Torres, Vanessa Schweitzer \\ Henry Ford Health System, Detroit, USA \\ E-mail: bmusgra1@hfhs.org \\ Received August 12, 2011; revised September 10, 2011; accepted September 24, 2011
}

\begin{abstract}
Ectopic odontomas are a rare disease process and need to be in the differential of the Otolaryngologist and Radiologist. Previous reports reveal the most common location to be the maxilla. We present a rare case of odontoma involving a turbinate causing nasal obstruction and foul-smelling rhinorrhea. Surgical excision is the treatment of choice with an endoscopic approach, if feasible.
\end{abstract}

Keywords: Odontoma, Turbinate, Ectopic, Complex, Compound, Dentin, Odontogenic, Rhinosinusitis

\section{Case Presentation}

A 54-year-old female presented with complaints of chronic right-sided nasal obstruction, rhinorrhea and a foul odor emanating from the nostril. The patient was otherwise healthy and reported only seasonal allergies and hypertension in her past medical history. On physical exam, there appeared to be a bony concretion in the floor of the right nasal cavity, impinging against the septal wall. A CT scan of the sinuses was performed and showed an expansile, centrally calcified lesion within the right nasal cavity involving the inferior turbinate and obstructing the inferior meatus (Figure 1 and 2). There

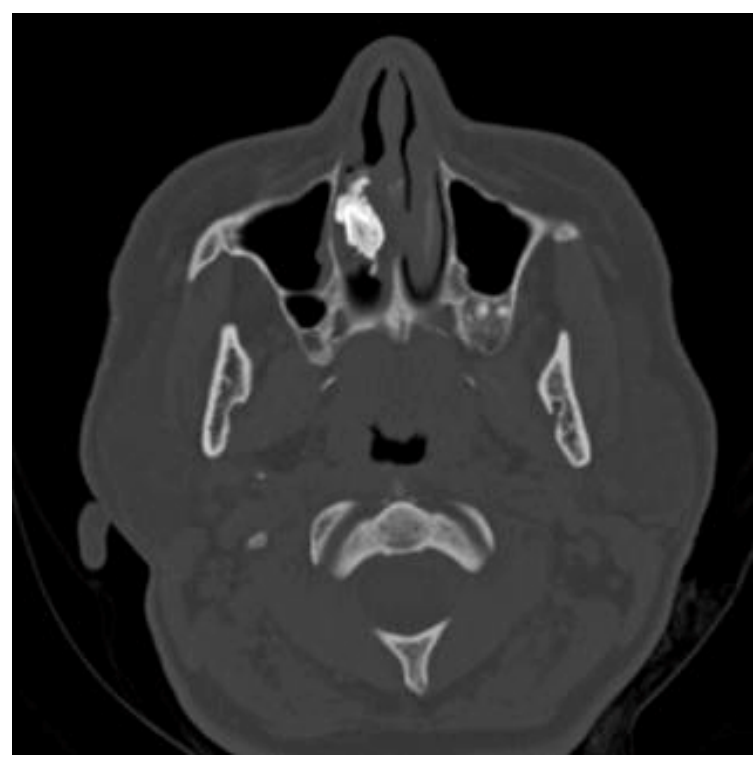

Figure 1. Axial CT Scan.



Figure 2. Coronal CT Scan.

was no bony destruction; however, there was thinning and possible erosion of the septum. There appeared to be no association to the underlying maxillary alveolar bone. The patient underwent endoscopic removal of the calcified mass and the histopathology contained two fragments of dentin (Figure 3 and 4). Admixed with the odontoma were partially calcified clusters of mixed flora including bacteria, fuso-spirochetes, and a few yeast for$\mathrm{ms}$, consistent with oral cavity type flora. The Gram stain was non-contributory because the tissue was previously decalcified. The AFB (Ziehl-Neelsen) stain was negative. 


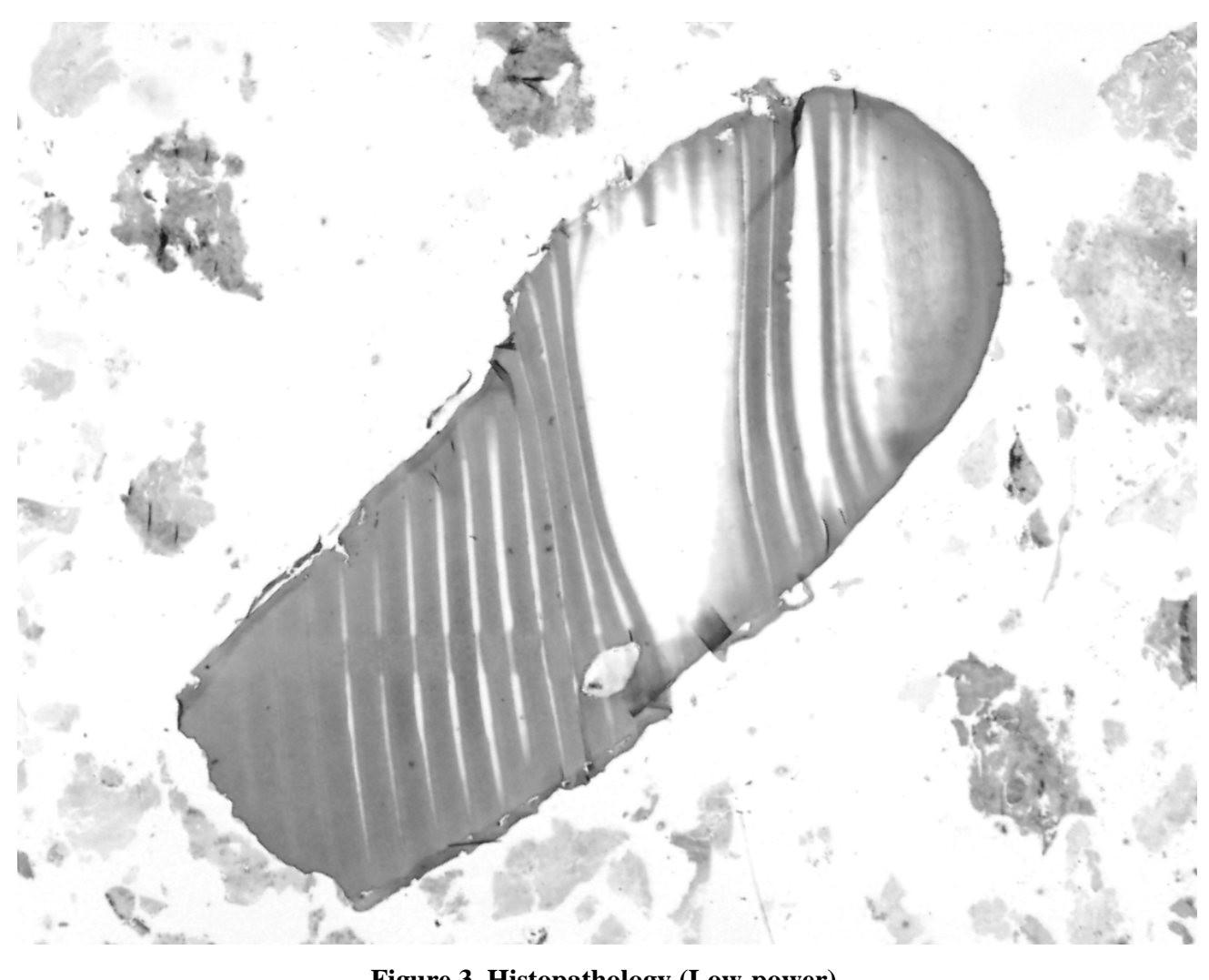

Figure 3. Histopathology (Low-power).



Figure 4. Histopathology (High-power). 


\section{Discussion}

Odontomas are the most common odontogenic tumor, containing both epithelial and mesenchymal elements such as enamel, dentin, pulp and cementum [1]. They are classified into two different categories: compound and complex. Compound odontomas are composed of enamel and dentin and form tooth-like structures. Complex odontomas are twice as prevalent and their histopathology is more haphazardly arranged [2]. The compound subtype appear as rudimentary toothlets on radiographs and are easily distinguishable, whereas the complex subtypes appear as radiopaque masses and require histopathology for diagnosis [3]. Odontomas in the sinuses or nasal cavity can be asymptomatic, or can manifest as rhinorrhea, malodor, nasal obstruction, or rhinosinusitis [4].

The radiographic assessment of our patient showed a radiopaque mass involving the right inferior turbinate. There did not appear to be any relationship to the maxillary alveolar tooth bearing bone. The histopathology of our specimen showed fragments of dentin as it was removed piecemeal. Nasal ectopic odontomas are extremely rare, and no prior documentation of an odontoma involving a turbinate was found in the literature. However, a case report involving two nasal floor odontomas with impacted incisors was published in 2003 [5]. The most common location for odontomas appears to be the maxilla, based on a retrospective review of 81 cases [6]. There has even been reported an ectopic odontoma in the middle ear, treated conservatively with observation and a BiCROS hearing aid for conductive hearing loss [7].

The differential diagnosis includes other odontogenic tumors such as cementomas, ameloblastic odontomas, Pinborg tumors, rhinolithiasis and osteosarcomas [8]. Treatment should include surgical resection when symptomatic, such as nasal obstruction in the above case. Endoscopic resection should be attempted when an odontoma involves the nasal cavity, but should be decided on a case-by-case basis due to the rarity of isolated involvement in the nose.

\section{References}

[1] S. G. Press, "Odontogenic Tumors of the Maxillary Sinus,” Current Opinion in Otolaryngology \& Head and Neck Surgery, Vol. 16, 2008, pp. 47-54. doi:10.1097/MOO.0b013e3282f419da

[2] M. Tomizawa, Y. Otsuka and T. Noda, "Clinical Observati- ons of Odontomas in Japanese Children: 39 Cases Including One Recurrent Case," International Journal of Paediatric Dentistry, Vol. 5, No. 1, 2005, pp. 37-43. doi:10.1111/j.1365-263X.2005.00607.X

[3] S. M. Lukes and K. M. Wachter, "Compound Odontoma: A Case Study,” Journal of Dental Hygeine, Vol. 77, No. 1, 2003, pp. 47-49.

[4] F. Lee, "Endoscopic Extraction of an Intranasal Tooth: A Review of 13 Cases,” Laryngoscope, Vol. 111, 2001, pp. 1027-1031. doi:10.1097/00005537-200106000-00017

[5] P. Cozza, R. Gatto, A. Marino and M. Mucedero, "Case Report: Two Nasal Floor Compound Odontomas Associated with Impacted Maxillary Incisor,” European Journal of Paediatric Dentistry, Vol. 4, No. 2, 2003, pp. 99-102.

[6] J. Y. Chang, J. T. Wang, Y. P. Wang, B. Y. Liu, A. Sun and C. P. Chiang, "Odontoma: A Clinicopathologic Study of 81 Cases,” Journal of the Formosan Medical Association, Vol. 102, No. 12, 2003, pp. 876-882.

[7] J. L. Sun, L. C. Ford, B. M. Rasgon and B. I. Lewis, "Odontoma of the Middle Ear: Case Report with 25-Year Follow-up,” Archives of Otolaryngology Head \& Neck Surgery, Vol. 130, 2004, pp. 1223-1224. doi:10.1001/archotol.130.10.1223

[8] T. H. Leem and T. T. Kingdom, "Radiology Quiz Case," Archives of Otolaryngology Head \& Neck Surgery, Vol. 134, 2008, pp. 667-668. doi:10.1001/archotol.130.10.1223 